\title{
The Expression and Significance of XB130 in Skin Basal Cell Carcinoma
}

\author{
Huang Yingjiann ${ }^{1}$ Duan Qiqi ${ }^{2}$, Wang Ning ${ }^{3}$ and Zheng Yan $^{1^{*}}$ \\ ${ }^{1,2,3}$ Derpartment of Dermatology, the Second Affiliated Hospital, School of Medicine, Xi'an Jiaotong University, 710004, China
}

Received: June 12, 2019; Accepted: June 19, 2019; Published: June 20, 2019

*Corresponding author: Zheng Yan, PhD, Professor, Department of Dermatology, the Second Affiliated Hospital, School of Medicine, Xi'an Jiaotong University, 157 Xiwu Road, Shaanxi Xi'an, 710004, China, Tel:+8615319931238,E-mail: zenyan66@126.com

\begin{abstract}
Background: Skin basal cell carcinoma (BCC) is the most common skin cancer with an increasing incidence rate worldwide. $\mathrm{XB130}$ is a novel adaptor protein for intracellular signal transduction and it participates in cell proliferation, cell survival, cell migration and cell apoptosis. Studies have demonstrated that XB130 is involved in cancer initiation and progression; however, its expression and role in BCC have not been investigated.

Methods: Immunohistochemistry was carried out to detect the expression of XB130 at protein level in skin samples obtained from patients with skin basal cell carcinoma $(\mathrm{n}=40)$ and healthy donors $(n=35)$.

Results: In our study, we found that the staining of XB130 was mostly observed in cytoplasm. In normal skin tissues, XB130 was expressed almost in the whole layer of the epidermis. And the expression of XB130 in BCC was significantly down-regulated compared to normal skin.
\end{abstract}

Conclusions: The study suggested that XB130 is involved in the pathogenesis of BCC and it may serve as a therapeutic target of BCC.

Keywords: XB130; skin basal cell carcinoma;

\section{Abbreviations}

BCC: Basal cell carcinoma;

XB130/AFAP1L2: Actin filament associated protein 1 like 2;

IHC: Immunohistochemistry; SH2: Src homology 2;

SH3: Src homology 3; PH: Pleckstrin homology;

PI3K: Phosphatidylinositol-3-kinase;

Tks5: Tyrosine kinase substrate with five SH3 domains;

DMBA: Dimethylbenzanthracene;

TPA: 12-0-tetradecanoylphorbol-13-acetate;

KO: Knockout.

\section{Introduction}

Skin basal cell carcinoma (BCC) is the most common skin cancer with an increasing incidence rate worldwide [1]. Clinically, they tend to develop on the sun-exposed skin of elderly individuals with fair skin type and rarely on mucosa or palms and soles [2]. BCC typically presents as a shiny, pink- or flesh-colored solitary pearly papule or nodule with central ulceration [3]. Patients often give a history of crusting and bleeding. Pigmented nodular BCCs are more common in colored people and a characteristic feature of BCC in Asians is "the black pearly appearance" [4]. The complete etiology and pathophysiology still remain unclear, but ultraviolet radiations and genetic predisposition seem to be the main etiological factors. Most BCCs are slow-growing and rarely metastasize or cause death despite the fact that they are locally invasive and can be destructive [5]. As a result, the discovery of promising target and new treatment is required.

XB130, also named AFAPIL2 (Actin filament associated protein 1 like 2), is a novel adaptor protein located on chromosome 10q25.3 which encodes an 818 amino acid protein with a molecular weight of 130,000 [6]. It contains many molecularbinding domains allowing its protein-protein and protein-lipid interactions. It plays a critical role in the regulation of signal transduction and affects cell proliferation, survival, invasion and migration $[7,8]$. XB130 is highly expressed in human thyroid and spleen and moderately expressed in other organs, like brain, lung, liver, kidney and skin [9]. Several studies have shown that XB130 is involved in cancer initiation and progression, but its role in skin basal cell carcinoma remains largely unknown. In our study, immunohistochemistry was carried out to examine the expression levels of XB130 in tissue specimens from 40 patients with BCC, as well as from 35 normal human controls. The aim was to explore the role of XB130 in the occurrence and development of BCC.

\section{Materials and Methods}

\section{Human skin tissue collection}

A total of 75 paraffin-embedded skin tissues, including 40 BCC tissues (22 males and 18 females, age range $=42-68$ years, mean age 57.5 years) and 35 normal skin tissues (16 males and 19 females, age range $=37-70$ years, mean age 56.0 years) for IHC, were obtained between May 2018 and May 2019 from the tissue bank of the Department of Dermatology at the Second Affiliated Hospital of Xi'an Jiaotong University. Written informed consents were obtained from all patients before the study initiation, and 
an ethics approval was obtained from the Institutional Ethics Committee of Xi'an Jiaotong University.

\section{Immunohistochemistry}

AFAP1L2 polyclonal antibody (1:200; Proteintech) was applied as primary antibody. Immunohistochemistry was performed according to a standard staining procedure [10]. Two pathologists observed the results under a microscope independently. The staining results were quantified based on the following scoring system in a semi-quantitative manner. The staining color was graded as follows: colorless $=0$, light yellow $=1$, yellowish brown $=2$, chocolate brown $=3$. The cell positive rate was graded according to the percentage of positive cells as follows: $0-5 \%=0,6-25 \%=1,26-50 \%=2,51-75 \%=3,>75 \%=4$. Five fields of view were randomly selected under a 400 -fold microscope, and 100 tumor cells were counted for each field. The total score for each microscopic field was the product of two scores and the average score of five fields was taken as the final score for each slide. For statistical analysis, the final scoring criteria were: 0 for negative $(-)$; $1-4$ for weak positive $(+)$; 5 -8 for moderate positive $(++)$; $9-12$ for strong positive $(+++)$.

\section{Statistical Analysis}

The SPSS statistical package (SPSS, Chicago, IL, USA) was used for data analysis. The chi-square test was used for immunohistochemistry analysis. $\mathrm{P}<0.05$ was considered statistically significant.

\section{Results}

We used immunohistochemistry to examine the expression of XB130 at protein level in skin samples from patients with BCC $(n=40)$ and normal controls $(n=35)$. As shown in Figure 1-3, the staining of XB130 was mostly observed in cytoplasm. In normal skin control, XB130 was expressed in almost the whole layer of the epidermis in $88.57 \%$ (31/35, Figure 1) of samples. In BCC, XB130 expression was negative in 28 samples (Figure 2). It can only be found expressed in $30.00 \%(12 / 40)$ of the samples and the staining color was light yellow (Figure 3 ). The sample positive rate of XB130 in BCC was lower than that in normal skin tissues $\left(\chi^{2}=26.178, \mathrm{P}<0.001\right)$. The positive statistics of each group are shown in (Figure 4) and [Table 1]. Meanwhile, the expression intensity of XB130 in BCC was significantly decreased compared to normal skin $\left(\chi^{2}=43.047, \mathrm{P}<0.001\right)$.

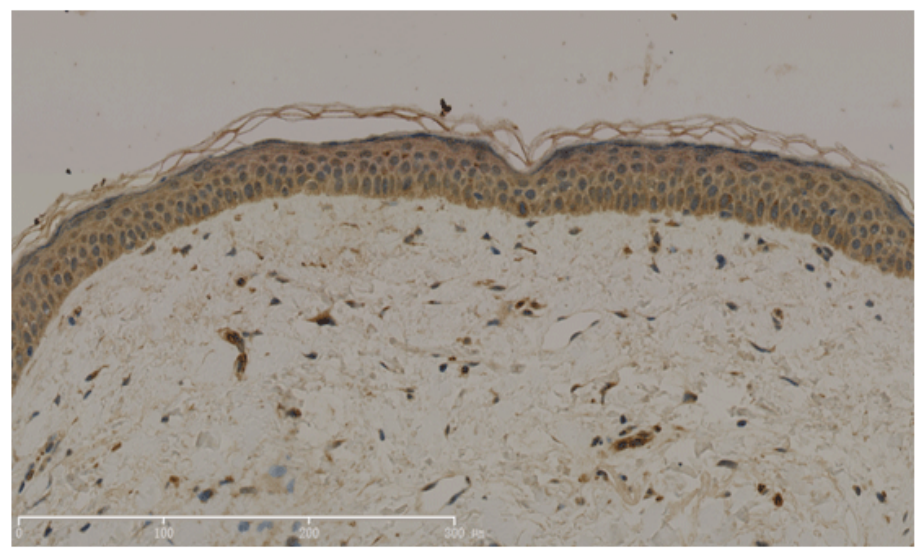

Figure 1: The expression of XB130 in normal skin $(\mathrm{SP} \times 200)$

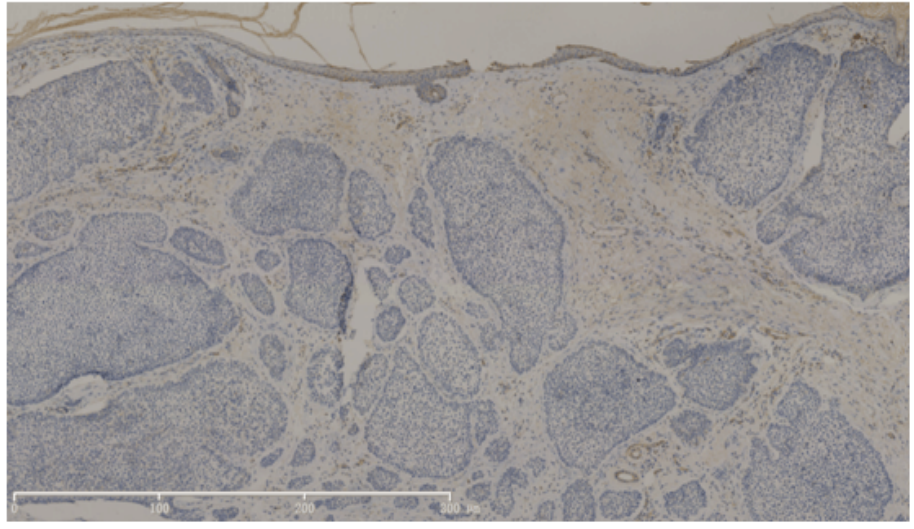

Figure 2: The negative expression of XB130 in BCC $(\mathrm{SP} \times 200)$ 


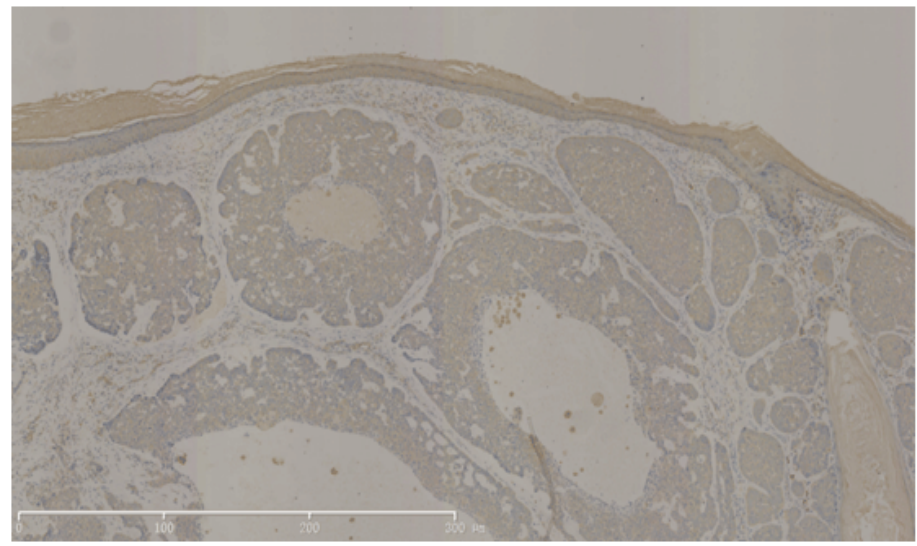

Figure 3: The positive expression of XB130 in BCC $(\mathrm{SP} \times 200)$

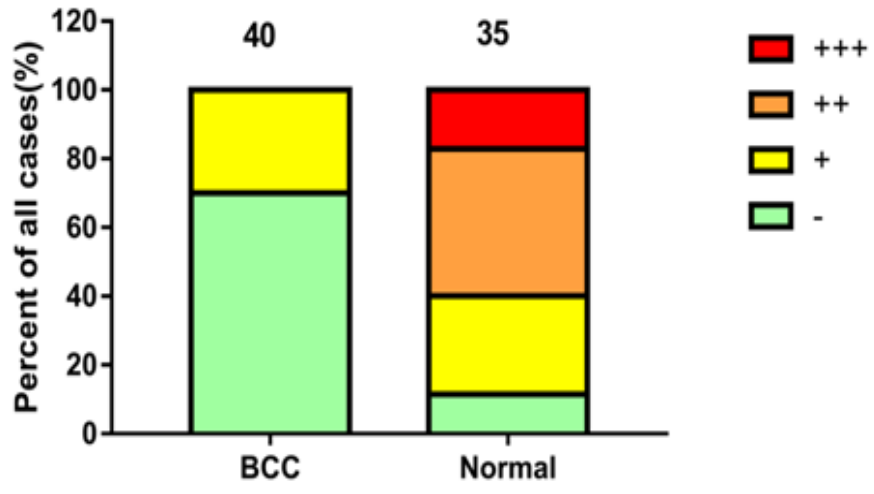

Figure 4: Semiquantitative analysis of XB130 staining

Table-1: Expression of XB130 in normal skin and BCC

\begin{tabular}{|c|c|c|c|c|c|}
\hline Group & n & \multicolumn{3}{|c|}{ Expression grade (n) } & Positive rate (\%) \\
\hline BCC & & - & + & ++ & +++ \\
\hline Normal skin & 40 & 28 & 12 & 0 & 0 \\
\hline Note: ${ }^{*}<0.001$, compared with normal skin
\end{tabular}

In conclusion, Semi-quantitative analysis of IHC results indicated that XB130 expression was remarkably down-regulated in BCC compared to the normal controls.

\section{Discussion}

BCC represents the most common skin malignancy. It usually occurs on sun-exposed areas like face and neck. The reported incidence rates per 100000 person-years are highest in Australia $(>1000 / 100000)$, lowest in Africans in Kenya $(<1 / 100000)$, 76.21 in England and 15-16.5 in Japan [5]. And the rates continue to increase worldwide probably due to the aging of the population and the frequent exposure to sunlight. Men are more frequently affected than women, usually after the age of 50 . However, a remarkable increase has been recently observed in young women, probably due to a higher use of tanning bed and the change of wearing style [2]. BCC is a complex disease, in which the interplay between ultraviolet radiation, phenotype and genotype fulfills a key role [3]. Other risk factors include ionizing radiations, arsenic ingestion and immune suppression. However, the mechanism has not been fully elucidated. The clinical manifestations are varied 
and skin biopsy is essential to confirm the diagnosis. Although they rarely causes death, multiple and recurrent BCCs severely affect the quality of life [11]. And the discovery of new molecular targets brings therapeutic promise.

Adaptor proteins are functional combinations of protein components that contain multiple modules but have no enzyme activity domain. They participate in the regulation of various signaling pathways. The action filament-associated protein (AFAP) family is an important member of the adaptor proteins, including AFAP1, AFAP1L1 and AFAP1L2/XB130 [7]. XB130 exhibits certain unique features.

XB130 contains molecular binding domains including SH2 and SH3 binding motifs and two $\mathrm{PH}$ domains that promote protein-protein and protein-lipid interactions [8]. For instance, as a substrate and mediator of multiple tyrosine kinases, XB130 can be phosphorylated and binds to the p85a subunit of phosphatidylinositol-3-kinase (PI3K), activating PI3K/Akt pathway $[12,13]$. The interaction between XB130 and scaffold protein Tks5 regulates Src-mediated cell proliferation [14,15] And there is XB130/Rac interation-dependent cell motility [16].

XB130 participates in various physiological and pathological procedures. It may play protective roles in many processes. For example, XB130 promotes bronchi alveolar stem cell proliferation in airway epithelial repair and regeneration $[17,18]$. Its deficiency delayed the recovery from systemic septic responses and acute lung injury [19].

The top-ranked disease associated with XB130 is cancer. XB130 may have dual roles as a tumor promoter or a tumor suppressor on a context-dependent manner. Some previous studies performed XB130 silencing on cancer cell lines using small interfering RNA and revealed that XB130 has a tendency to enhance cell proliferation, cell migration and invasion, indicating its oncogenic properties [20-23]. Several studies have demonstrated the relationship between the expression levels of XB130 and the tumor classification, lymph node metastasis and prognosis, but the results differ among tumor types. XB130 has showed oncogenic properties in prostate cancer [21], esophageal cancer [24], pancreatic ductal adenocarcinoma [25], breast cancer[22], with high expression levels predicting a short survival time and a poor prognosis, whereas the reverse is true for gastric cancer. A study showed that although XB130 could be used as a predictor for gastric cancer due to its high expression, the low expression indeed indicates a poor prognosis [26]. XB130 also showed a tumor suppressive trait in papillary thyroid carcinoma because its expression was significantly lower than that in normal thyroid tissue and benign lesions [27].

A recent study used XB130-deficient mice to investigate the role of XB130 in carcinogen-induced skin tumorigenesis. The study indicated tumor-suppressive functions of XB130. DMBA/TPA-treated XB130 KO male mice developed a higher number of skin tumors including dysplasia, verrucous dysplasia and keratoacanthoma [28]. Deficiency of XB130 dramatically increased epidermal tumor cell proliferation and enhanced the inflammatory response with accumulation of infiltrating neutrophils and inflammatory cytokines.

In conclusion, the findings from our study showed that the XB130 expression was down-regulated in BCC compared with normal skin. XB130 could be of great importance in the tumorigenesis of BCC, which makes it a potential therapeutic target. It may serve as a tumor suppressor and further study is required in order to unravel the specific mechanism. Understanding the expression and significance of XB130 may help to manage this multifaceted disease.

\section{Acknowledgements}

This work was supported by grants from the National Natural Science Foundation of China (81573055).

\section{References}

1. Verkouteren JAC, Ramdas KHR, Wakkee M, Nijsten T. Epidemiology of basal cell carcinoma: scholarly review. Br J Dermatol. 2017; 177(2):359-372. doi:10.1111/bjd.15321

2. Pellegrini C, Maturo MG, Di Nardo L, Ciciarelli V, Gutierrez GarciaRodrigo C,Fargnoli MC. Understanding the Molecular Genetics of Basal Cell Carcinoma. Int J Mol Sci. 2017;18(11). doi:10.3390/ijms18112485

3. Rubin AI, Chen EH,Ratner D. Basal-cell carcinoma. N Engl J Med. 2005;353(21):2262-9. doi:10.1056/NEJMra044151

4. Higgins S, Nazemi A, Chow M,Wysong A. Review of Nonmelanoma Skin Cancer in African Americans, Hispanics, and Asians. Dermatol Surg. 2018;44(7):903-910. doi:10.1097/DSS.0000000000001547

5. Tanese K. Diagnosis and Management of Basal Cell Carcinoma. Curr Treat Options Oncol. 2019;20(2):13. doi:10.1007/s11864-019-06100

6. Xu J, Bai XH, Lodyga M, et al. XB130, a novel adaptor protein for signal transduction. J Biol Chem. 2007;282(22):16401-12. doi:10.1074/jbc. M701684200

7. Bai XH, Cho HR, Moodley S, Liu M. XB130-A Novel Adaptor Protein: Gene, Function, and Roles in Tumorigenesis. Scientifica (Cairo). 2014; 2014:903014. doi:10.1155/2014/903014

8. Takeshita H, Shiozaki A, Bai XH, et al. XB130, a new adaptor protein, regulates expression of tumor suppressive microRNAs in cancer cells. PLoS One. 2013;8(3):e59057. doi:10.1371/journal.pone.0059057

9. Zhang R, Zhang J, Wu Q Meng F,Liu C. XB130: A novel adaptor protein in cancer signal transduction. Biomed Rep. 2016; 4(3):300-306. doi:10.3892/br.2016.588

10. Pinheiro C, Longatto-Filho A, Scapulatempo C, et al. Increased expression of monocarboxylate transporters 1,2 , and 4 in colorectal carcinomas. Virchows Arch. 2008;452(2):139-146. doi:10.1007/ s00428-007-0558-5

11. Puig S,Berrocal A. Management of high-risk and advanced basal cell carcinoma. Clin Transl Oncol. 2015;17(7):497-503. doi:10.1007/ s12094-014-1272-9

12. Shiozaki A, Shen-Tu G, Bai X, et al. XB130 mediates cancer cell proliferation and survival through multiple signaling events downstream of Akt. PLoS One. 2012;7(8):e43646. doi:10.1371/ journal.pone.0043646

13. Lodyga M, De Falco V, Bai XH, et al. XB130, a tissue-specific adaptor protein that couples the RET/PTC oncogenic kinase to PI 3-kinase 
pathway. Oncogene. 2009;28(7):937-49. doi:10.1038/onc.2008.447

14. Moodley S, Hui Bai X, Kapus A, Yang B,Liu M. XB130/Tks5 scaffold protein interaction regulates Src-mediated cell proliferation and survival. Mol Biol Cell. 2015;26(24):4492-502. doi:10.1091/mbc.E1507-0483

15. Moodley S, Derouet M, Bai XH, et al. Stimulus-dependent dissociation between XB130 and Tks5 scaffold proteins promotes airway epithelial cell migration. Oncotarget. 2016;7(47):76437-76452. doi:10.18632/ oncotarget.13261

16. Lodyga M, Bai XH, Kapus A,Liu M. Adaptor protein XB130 is a Raccontrolled component of lamellipodia that regulates cell motility and invasion. J Cell Sci. 2010; 123(Pt 23):4156-69. doi:10.1242/jcs.071050

17. Zhao J, Wang Y, Wakeham A, et al. XB130 deficiency affects tracheal epithelial differentiation during airway repair. PLoS One. 2014; 9(10):e108952. doi:10.1371/journal.pone.0108952

18. Toba H, Wang Y, Bai X, et al. XB130 promotes bronchioalveolar stem cell and Club cell proliferation in airway epithelial repair and regeneration. Oncotarget. 2015;6(31):30803-17. doi:10.18632/ oncotarget.5062

19. Toba H, Tomankova T, Wang Y, et al. XB130 deficiency enhances lipopolysaccharide-induced septic response and acute lung injury. Oncotarget. 2016;7(18):25420-31. doi:10.18632/oncotarget.8326

20. Li GM, Liang CJ, Zhang DX, Zhang LJ, Wu JX,Xu YC. XB130 Knockdown Inhibits the Proliferation, Invasiveness, and Metastasis of Hepatocellular Carcinoma Cells and Sensitizes them to TRAILInduced Apoptosis. Chin Med J (Engl). 2018;131(19):2320-2331. doi:10.4103/0366-6999.241800
21. Chen B, Liao M, Wei Q, et al. XB130 is overexpressed in prostate cancer and involved in cell growth and invasion. Oncotarget. 2016; 7(37):59377-59387. doi:10.18632/oncotarget.11074

22. Li J, Sun W, Wei H, Wang X, Li H,Yi Z. Expression of XB130 in human ductal breast cancer. Int J Clin Exp Pathol. 2015; 8(5):5300-5308.

23. Shi M, Zheng D, Sun L, et al. XB130 promotes proliferation and invasion of gastric cancer cells. J Transl Med. 2014; 12:1. doi:10.1186/14795876-12-1

24. Shiozaki A, Kosuga T, Ichikawa D, et al. XB130 as an independent prognostic factor in human esophageal squamous cell carcinoma. Ann Surg Oncol. 2013; 20(9):3140-3150. doi:10.1245/s10434-012-24744

25. Zhang J, Jiang X, Zhang J. Prognostic significance of XB130 expression in surgically resected pancreatic ductal adenocarcinoma. World J Surg Oncol. 2014;12:49. doi:10.1186/1477-7819-12-49

26. Shi M, Huang W, Lin L, et al. Silencing of XB130 is associated with both the prognosis and chemosensitivity of gastric cancer. PLoS One. 2012;7(8):e41660. doi:10.1371/journal.pone.0041660

27. Shiozaki A, Lodyga M, Bai XH, et al. XB130, a novel adaptor protein, promotes thyroid tumor growth. Am J Pathol. 2011;178(1):391-401. doi:10.1016/j.ajpath.2010.11.024

28. Cho HR, Wang Y, Bai X, et al. XB130 deficiency enhances carcinogeninduced skin tumorigenesis. Carcinogenesis. 2019. doi:10.1093/ carcin/bgz042 